\title{
Cuidado às mulheres em situação de violência conjugal: importância do psicólogo na Estratégia de Saúde da Família'
}

\author{
Nadirlene Pereira Gomes ${ }^{\mathrm{a}}{ }^{*}$, Alacoque Lorenzini Erdmann ${ }^{\mathrm{b}}$, Karine Patrícia Stulp $^{\mathrm{b}}$, Normélia Maria Freire \\ Diniz a , Cíntia Mesquita Correiac , Selma Regina de Andrade ${ }^{b}$ \\ ${ }^{a}$ Escola de Enfermagem da Universidade Federal da Bahia, Salvador, BA, Brasil \\ ${ }^{b}$ Escola de Enfermagem da Universidade Federal de Santa Catarina, Florianópolis, SC, Brasil \\ 'Escola Bahiana de Medicina e Saúde Pública, Salvador, BA, Brasil
}

\begin{abstract}
Resumo: A violência conjugal compromete a saúde mental das mulheres, estando associada à baixa autoestima $\mathrm{e}$ depressão. No fomento de ações para enfrentamento desse agravo, destaca-se a atuação dos psicólogos. Objetivou-se compreender o significado do apoio psicológico à mulher em situação de violência conjugal, no âmbito da Estratégia de Saúde da Família. Foram realizadas entrevistas com 52 profissionais que atuam em unidades de saúde em Santa Catarina, Brasil. A coleta e análise de dados basearam-se na Teoria Fundamentada nos Dados. Os profissionais de saúde significam que as mulheres em vivência de violência conjugal necessitam de apoio psicológico e as referenciam para o psicólogo, por considerá-los mais bem preparados para o empoderamento da mulher no sentido de romper com a situação de violência. Todavia, queixam-se do número limitado de psicólogos, considerando a demanda na comunidade. Sinaliza-se para a necessidade de repensar as ações em saúde no âmbito da Atenção Primária à Saúde, a partir da integração e articulação intersetorial a fim de que seja assegurado às mulheres o apoio psicológico necessário para o enfrentamento da violência conjugal.

Palavras-chave: violência contra a mulher, violência na família, saúde da família, psicologia.
\end{abstract}

\section{Introdução}

Entende-se por violência contra a mulher qualquer ação, baseada na desigualdade de gênero, que cause à mulher dano patrimonial, moral, psicológico, físico e/ou sexual (Brasil, 2006). Tais situações comprometem a saúde física, mental e o desenvolvimento humano das mulheres, sendo retratadas no perfil de morbi-mortalidade feminina.

No que diz respeito às taxas de homicídios femininos, dados da Organização Mundial de Saúde (OMS), referentes aos anos de 2006 a 2010, apontam que o Brasil ocupa a sétima posição dentre 84 países. Com relação à morbidade, estima-se que os custos do Sistema Único de Saúde (SUS) comprometem cerca de $5 \%$ de todo o Produto Interno Bruto (PIB) (Brasil, 2008). Somente em 2011, foram registrados 73.633 atendimentos relativos à Violência Doméstica, Sexual e/ou outras Violências pelo Sistema de Informação de Agravos de Notificação (SINAN). Destes, $65,4 \%$ foram prestados a mulheres, sendo a residência o local mais prevalente e o cônjuge ou ex- cônjuge os principais autores da violência (Waisenfisz, 2011), alertando para a problemática da violência doméstica, na qual se insere a violência conjugal. Vale referir ainda que, sobretudo por conta do mascaramento que permeia a violência que se dá

1 Recorte do trabalho de pós-doutorado intitulado "Mulheres em situação de violência conjugal: construindo práticas de cuidado de enfermagem e saúde na ESF" - Apresentado à Universidade Federal de Santa Catarina. Florianópolis, SC, Brasil.

* E-mail para correspondência: nadirlenegomes@hotmail.com no espaço da casa, o número de mulheres em vivência de violência ainda não é conhecido, o que acaba por camuflar a magnitude do complexo fenômeno.

A violência conjugal ancora-se na violência de gênero, estando esta alicerçada no sistema patriarcal, que naturaliza a supremacia masculina atribuindo socialmente às mulheres o papel de submissão e obediência ao homem/ esposo e de dona-de-casa, devendo ser responsável pelo cuidado com a casa, filho e marido (Dias, 2007; Gomes, 2009). Essa construção social de gênero, que remete a mulher ao âmbito privado, nos permite compreender a dificuldade desta em perceber-se em vivência de violência no espaço doméstico, bem como sua permanência na relação. Além disso, não podemos deixar de considerar que a "violência conjugal baixa a autoestima, interfere negativamente na qualidade de vida e pode levar à morte, mas quase sempre essa morte é simbólica, porque magoa a alma, destrói os sonhos, rouba a esperança e a vontade de viver" (Gomes, 2009, pp. 142-143). Percebe-se, pois a importância do apoio psicológico para as mulheres nessa situação.

Fica claro ainda que as marcas da violência não se limitam as feridas visíveis. Esse agravo vai definhando aos poucos as defesas da mulher, que muitas vezes se sente culpada pela violência sofrida. A somatização de tal vivência compromete a saúde mental das mulheres, estando associada ao isolamento, medo, ansiedade, baixa autoestima, depressão, estresse pós-traumático, ideação suicida e a ten- 
tativa de suicídio (Maia et al., 2001). Tal quadro reafirma a necessidade de profissionais na área de psicologia para melhor suporte às mulheres.

No âmbito da Atenção Primária à Saúde (APS), recomenda-se na Estratégia Saúde da Família (ESF) a presença de pelo menos um profissional da área de saúde mental. Em muitos municípios, o psicólogo integra o Núcleo de Apoio à Saúde da Família (NASF), com o objetivo de ampliar a abrangência e a resolubilidade das ações da atenção básica, apoiando as ações desenvolvidas pela ESF, tanto assistencial, quanto de suporte técnico-pedagógico às equipes de referência (Blanques, 2010; Brasil, 2008; Campos \& Domitti, 2007).

Com relação à finalidade dos atendimentos realizados por psicólogos no âmbito da ESF, destaca-se a importância no fomento de ações para prevenção e diminuição dos agravos referentes à violência doméstica. A principal é o empoderamento das mulheres no que tange ao processo mais amplo de resgate e fortalecimento da autoestima da mulher, que na dimensão individual expressa à valorização de si. O termo empowerment corresponde ao fortalecimento das mulheres a fim de redistribuição do poder em favor delas, o que remete a mudanças sociais e culturais, tais quais as relações de poder desiguais de gênero e iniquidades sociais (Hanada, D'Oliveira, \& Schraiber, 2008).

Assim sendo, torna-se imprescindível a presença de profissionais preparados para prestar suporte psicológico às mulheres em situação de violência conjugal, motivando-nos a propor o seguinte questionamento: Quais os significados que os profissionais que atuam na ESF atribuem à importância das ações do psicólogo no cuidado à mulher em situação de violência conjugal? Elegeu-se como objetivo compreender o significado do apoio psicológico à mulher em situação de violência conjugal, no âmbito da Estratégia de Saúde da Família.

\section{Metodologia}

Trata-se de um estudo realizado em um município de Santa Catarina, Brasil, vinculado ao projeto de pós-doutoramento intitulado "Mulheres em situação de violência conjugal: construindo práticas de cuidado de enfermagem e saúde na ESF", financiado pela FAPESB. A pesquisa contou com 16 equipes de saúde da família que integram os cinco centros de saúde de um determinado Distrito Sanitário. A escolha do distrito sanitário foi motivada pela composição de comunidades com contextos socioeconômicos distintos, podendo assim representar melhor a realidade do cuidado à mulher em situação de violência conjugal do município.

$\mathrm{O}$ projeto de pesquisa foi submetido e aprovado pelo Comitê de Ética em Pesquisa da Universidade Federal de Santa Catarina (Parecer No 21560/2012). Aos sujeitos da pesquisa foram esclarecidos a relevância e o objetivo do estudo, dentre outros aspectos éticos com base na Resolução 196/96. Solicitou-se a assinatura do Termo de Consentimento Livre e Esclarecido.
O processo de coleta e análise de dados seguiu o método da Teoria Fundamentada nos Dados (TFD) que possibilita a identificação, o desenvolvimento e a relação de conceitos, elaborados a partir de situações e sujeitos com características e práticas diferenciadas (Strauss \& Corbin, 2008). O critério de Amostragem Teórica definiu três grupos amostrais (GA). $\mathrm{O} 1^{\circ} \mathrm{GA}$ foi composto por 17 técnicos de enfermagem, 13 enfermeiros e 12 médicos que atuam nas 16 equipes de Saúde da Família. O $2^{\circ}$ GA foi constituído por dois psiquiatras, dois psicólogos e uma assistente social que atuam junto ao NASF do distrito em estudo. Finalmente, o $3^{\circ} \mathrm{GA}$ foi composto por cinco coordenadores de saúde, responsáveis pelas Unidades de Saúde. Realizou-se, portanto 52 entrevistas, desenvolvidas em encontros individuais, com autorização para gravação.

As entrevistas foram transcritas e analisadas conforme pressupostos da TFD, a partir do processo de codificação aberta, axial e seletiva, tomando como base os cinco conceitos estruturais: contexto, condição causal, condição interveniente, estratégias e consequências (Strauss \& Corbin, 2008). O processo de organização dos dados foi realizado com auxílio do software NVivo $8.0^{\circledR}$. As falas foram apresentadas sem identificação dos sujeitos, a fim de garantir a confidencialidade das informações, de modo que foram identificados por meio da inicial maiúscula da categoria profissional, ou letra $\mathrm{C}$ se coordenador, seguida por um numeral arábico.

Vale referir que o processo de codificação resultou o fenômeno "Reconhecendo a violência conjugal como problema de saúde pública e a necessidade de gestão do cuidado à mulher". Realizou-se validação do modelo teórico com profissionais de saúde das unidades entrevistadas e com dez pesquisadores com experiência na TFD.

\section{Resultados}

As categorias aqui apresentadas expressam conceitos relacionados à atuação do psicólogo, que atua no âmbito da ESF, para o cuidado à mulher em situação de violência conjugal. Esses conceitos articulam-se e interagem permeando os cinco conceitos estruturais do modelo. São elas:

\section{Relacionando violência conjugal e a necessi- dade de apoio psicológico para mulher e filhos}

Os profissionais da ESF, inclusive as psicólogas que atuam no NASF, significam que as mulheres em vivência de violência conjugal necessitam de apoio psicológico em decorrência de problemas emocionais. As crianças inseridas em um lar permeado pela violência também apresentam comprometimento de sua saúde mental, com implicações para seu desenvolvimento humano. Tais conceitos encontram-se ilustrados nas subcategorias a seguir: 


\section{Defendendo que a mulher precisa de suporte psicológico}

Para os profissionais que atuam na ESF, mulheres em vivência de violência conjugal necessitam de apoio psicológico para empoderá-las no sentido de buscar saídas para uma vida livre de violência, conforme ilustram as falas:

"Muitas mulheres que sofrem isso não saem dessa situação por medo de não ter onde se apoiar. . . . se a gente conseguisse apoiar psicologicamente, acho que seria uma boa forma de ajudar". (TE6).

"Eu vejo que na situação de violência tem que ter apoio psicológico... para ela se livrar disso". (M1).

Os psicólogos que atuam no NASF confirmam a importância do apoio psicológico para as mulheres em vivência de violência. Suas falas nos permitem compreender melhor o comprometimento emocional:

"Há uma vinculação de dependência: de que o outro vai lhe suprir em alguma coisa. E ela acha que não vai dar conta de viver sozinha. Eu consigo perceber a dificuldade em desenvolver uma alta autoestima" (Pc1).

"É como se ela se acostumasse a uma situação negativa. . . ela vive o problema, mas não chegou naquele ponto de buscar uma ajuda". (Pc2).

\section{Defendendo que a criança precisa de suporte psicológico}

Crianças que presenciam a violência conjugal encontram-se vulneráveis ao comprometimento de sua saúde mental, tendendo a naturalizar e reproduzir tal comportamento nas relações sociais, inclusive posteriormente quando em uma relação de conjugalidade. É o que revelam as falas a seguir:

"A criança que assiste a mãe apanhar tem que ter um apoio psicológico bom, porque ela vai levar aquilo para vida. Ela vai entender que tudo se resolve com agressão ... . não só com a esposa, mas também com o colega da rua, da escola". (TE1).

"São crianças que vão tendo problemas psicológicos por causa do que estão vivenciando em casa. . . . elas chegam aqui acuadas, meio reprimidas. Às vezes, não conseguem acompanhar o colégio por causa disso". (M10).

\section{Referindo sobre a importância do psicólogo na ESF}

Diante a vivência de violência conjugal pela mulher e a necessidade de empoderamento para o rompimento da relação ancorada no desrespeito e na violência, os profissionais entrevistados informam referenciamento dos casos identificados para o psicólogo que integra o NASF. As subcategorias ilustram os significados atribuídos pelos profissionais no que tange a importância do psicólogo para o empoderamento da mulher no sentido de fortalecê-la para o rompimento da relação de violência.

\section{Acreditando que o psicólogo está mais preparado}

Os profissionais que integram a equipe de referência justificam que os encaminhamentos para os psicólogos do NASF se dão por considerá-los mais preparados para abordagem da mulher, conforme ilustram as falas a seguir:

"Se eu perceber ou achar que ela quer falar alguma coisa . . o o mais correto era fazer essa abordagem junto com a psicologia. ... porque tem que ser uma abordagem correta para que ela não saia e nunca mais volte". (E13).

"o psicólogo é a pessoa mais adequada para fazer essa abordagem e que talvez pudesse provocar uma mudança nesse comportamento. Acho que a gente serve como um apoio, mas a ação do psicólogo é mais efetiva". (E7).

\section{Elencando a contribuição do apoio psicológico}

O apoio psicológico, conforme significam os profissionais, viabiliza o fortalecimento da mulher no sentido de traçar saídas para a relação conjugal pautada na violência. As falas revelam as contribuições da atuação do psicólogo para as mulheres em situação de violência:

"existe a necessidade de um apoio psicológico mesmo, na expectativa de ajudá-la a se localizar na situação, de conseguir ver de outro ângulo, conseguir sair da situação de vítima da violência". (M1).

"Eu busco mobilizá-la, sensibilizá-la ... fortalecendo-a para que consiga pensar, fortalecendo as suas posturas". (Pc2).

\section{Queixando-se do número de psicólogo na ESF}

Para os profissionais entrevistados, há uma dificuldade para suprir as demandas relacionadas a problemas emocionais /psicológicos no âmbito da ESF, sendo necessária maior disponibilidade de psicólogos. Tais considerações foram agrupadas nas subcategorias a seguir:

\section{Reconhecendo que o número de psicólogos não atende a demanda}

Ao identificarem mulheres em situação de violência conjugal, os profissionais que compõem a equipe de referência encaminham-nas para o psicólogo do NASF. No entanto, diante da grande demanda, os entrevistados queixam-se da limitada disponibilidade do profissional:

"eu trabalho em três unidades. A gente tem violência contra a mulher, violência contra o idoso, violência contra adolescente e a gente tem milhões de fatores que a gente não consegue dar a intensidade de assistência que essas pessoas necessitam". ( $\mathrm{Pcl})$.

"Às vezes tem que remanejar uma situação que está mais estabilizada para fazer pelo menos um primeiro atendimento com outra pessoa. É difícil para as psicólogas”. (C4). 


\section{Percebendo a deficiência nas ações de promoção à saúde}

Concordando sobre o limitado número de psicólogos na ESF, os psicólogos que atuam no NASF acrescentam que sua atuação nesse contexto contradiz os pressupostos da ESF, uma vez que não conseguem realizar ações de promoção à saúde e prevenção à agravos.

"a gente fica se dividindo e a demanda é muito grande. ... a gente não consegue atender com a frequência e com a intensidade que deveria. E o NASF tem aquela proposta de apóio a ESF, e não só de assistência. A proposta é de promoção. Mas não se faz nem uma coisa nem outra. Acaba frustrando". (Pc2).

"às vezes tem mais trabalho curativo que preventivo". (Pc1).

\section{Discussão}

No contexto de violência conjugal, percebe-se uma relação de dependência emocional da mulher ao seu cônjuge, expressa, por exemplo, pela ideia de que não vive sem o outro. A dependência emocional, desvelada nesse estudo, pode ser entendida como um padrão persistente de necessidades psicológicas que não foram atendidas, sendo então projetadas em relacionamentos futuros. Pode estar associada ainda à necessidade excessiva que uma pessoa sente pela outra. Em se tratando da temática violência contra a mulher encontram-se presentes, além da necessidade do outro, características como carência de autoestima e idealização do outro (Blasco, 2000; Silva, Falbo Neto \& Cabral Filho, 2009).

Dependentes emocionalmente do cônjuge, muitas mulheres sequer percebem-se em vivência de violência. Outras não conseguem reagir diante da situação e permanecem na relação mesmo com muito sofrimento sem, contudo, buscarem meios de romper com a violência conjugal. Estudiosos apontam que o silêncio pode ser pensado como uma estratégia de adaptação para limitar a violência do parceiro e preservar o casamento e a família, papel para o qual se preparou durante toda a vida. Assim sendo, a perspectiva de romper com a relação pode vir a ser, para a mulher, mais temível que a própria violência (Gomes, 2009; Hirigoyen, 2006). Toda essa conjuntura desvela a importância do apoio psicológico às mulheres em situação de violência. $\mathrm{O}$ estudo também mostra a necessidade de apoio emocional para filhos de casais que presenciam a violência conjugal, o que corrobora com achados de outros estudos que afirmam inclusive o comprometimento para o desenvolvimento humano das crianças. Estas podem apresentar déficits cognitivos, baixa autoestima, quadros depressivos, baixo rendimento escolar e comportamentos inadequados (Day, 2003).

Considerando as implicações da violência conjugal mencionadas, são essenciais estratégias de empoderamento para que as mulheres possam buscar uma vida sem violência para si e seus filhos. Nesta perspectiva, enfermeiros e médicos que atuam nas equipes de saúde, ao identificarem ou suspeitarem da vivência de violência pelas mulheres, referenciam-nas para apoio psicológico junto ao NASF. Para os entrevistados, a atuação do profissional de psicologia é essencial no processo de empoderamento da mulher no sentido de perceber-se na relação como sujeito capaz de traçar seu caminho, de enxergar outras possibilidades. O acompanhamento psicológico à mulher faz-se necessário a fim de auxiliá-la a encontrar formas de se reestruturar emocionalmente, recuperar sua autoestima e autoconfiança, e assim possibilitar lidar com as situações de crise e pensar estratégias de convívio mais saudável em suas relações (Maia et al., 2001). O diferencial da psicologia consiste justamente na valorização das potencialidades da mulher e também na capacidade de fomentar o "não dito" (Nepomuceno \& Brandão, 2011). O processo de empoderamento deve contemplar a construção de uma autoimagem e confiança positiva e o desenvolvimento de habilidades para o pensamento crítico, para a tomada de decisões e para a ação (Stromquist, 2005). Deve-se, portanto, assumir a Psicologia como ciência sabedora dos processos relacionados aos indivíduos, seus modos de pensar e agir (Rose, 2008).

No que tange a violência, estudos sustentam que esta é compreendida pelos profissionais, sobretudo os de medicina e de enfermagem, como pertencente ao campo de saúde mental, havendo assim um maior direcionamento das mulheres para psicólogos (D'oliveira, 2000; Kiss, 2004; Pedrosa, 2003; Schraiber; D’oliveira; França-Junior $\&$ Pinho, 2002). Nosso estudo corrobora tal achado ao apontar que enfermeiros e médicos das equipes de saúde referenciam as mulheres em situação de violência conjugal para os psicólogos do NASF. Tal achado sinaliza para a dificuldade dos profissionais em geral de lidar com a dimensão interpessoal e com o sofrimento alheio (Hanada et al., 2008). Por outro lado, nos conduz à ideia do psicólogo como profissional da subjetividade, que sabe lidar com 0 sofrimento, as angústias e os anseios do outro. Faz-nos ainda refletir sobre a crença de que cabe somente ao psicólogo desenvolver ações em que se necessita de um olhar mais profundo sobre os sujeitos. Essa visão atrela-se à velha concepção do psicólogo clínico, cujas atividades tradicionais ainda se mostram enraizadas no modelo clínico-biomédico (Freire \& Pichelli, 2013).

O estudo revela ainda que, diante dos encaminhamentos para o psicólogo do NASF, estes não conseguem atender as demandas da comunidade, de modo que, para os entrevistados, sua atuação não condiz com as premissas do modelo de APS, que preza por ações de promoção da saúde e prevenção de doenças e agravos, em que se insere a violência contra a mulher. Neste contexto, os profissionais de saúde entrevistados defendem maior disponibilidade de psicólogos no âmbito da ESF.

Todavia, é importante que os espaços de saúde busquem maior interação e articulação com demais serviços para apoio psicológico, sobretudo a partir da interdisciplinaridade e da intersetorialidade, conforme orienta a APS. É importante salientar que, enquanto estratégia de organi- 
zação das novas práticas de cuidado e de gestão em saúde, o NASF deve desempenhar suas ações de forma compartilhada com as equipes de referências, visando à construção e ativação de espaços para comunicação, compartilhamento de conhecimentos e corresponsabilização dos casos com a ESF (Campos \& Domitti, 2007). Além disso, o acolhimento e a escuta se relacionam à humanização do atendimento e, portanto dizem respeito a todos os profissionais que prestam assistência direta à clientela.

\section{Consideracões finais}

Problemas emocionais, como a dependência do outro, apresentados por mulheres em vivência de violência conjugal justificam a necessidade de apoio psicológico, conforme significam os entrevistados. Diante da suspeita ou do reconhecimento de vivência de violência conjugal pela mulher, os profissionais de saúde as referenciam para o psicólogo que atua no NASF. Todavia, considerando as diversas necessidades no âmbito da ESF, os próprios psicólogos referem dificuldades para atender a demanda, inclusive para realizar ações de promoção da saúde e prevenção do agravo, o que contradiz as premissas da estratégia de saúde em que se encontram inseridos.

A necessidade de mais psicólogos disponíveis na ESF foi defendida por profissionais da enfermagem, medicina e também da psicologia. Vale ressaltar, entretanto, que a promoção de saúde se constrói a partir de ações interdisciplinares e intersetoriais, a fim de que seja assegurado às mulheres o apoio psicológico necessário para o enfrentamento da violência conjugal. Nesse caso, é essencial maior integração para a formação de uma rede de serviços, de diversas áreas, que sejam corresponsáveis pela melhoria da qualidade de vida dos usuários.

\title{
Care for women in situations of domestic violence: Importance of the psychologist in the Family Health Strategy
}

\begin{abstract}
Domestic violence damages the mental health of women and is associated with low self-esteem and depression. In promoting actions to deal with this injury, there is the work of psychologists. This study aimed to understand the meaning of psychological support to women in situations of domestic violence within the Family Health Strategy. It was held interviews with 52 professionals working in health units in Santa Catatina, Brazil. The collection and analysis of data were based on Grounded Theory. Health professionals mean that women experience of domestic violence need psychological support and refer to the psychologist, considering them better prepared for the empowerment of women in order to break with the violence. However, complain about the limited number of psychologists, considering the demand in the community. Signals to the need to rethink health actions in the context of Primary Health Care, from the integration and intersectoral processes so that women needed psychological support for coping with domestic violence is ensured.
\end{abstract}

Keywords: violence against women, violence in the family, family health, psychology.

\section{Les Soins pour les femmes dans les situations de violence conjugale: L'importance du psychologue dans la Stratégie de santé familiale}

Résumé: La violence domestique compromet la santé mentale des femmes et est associée à une faible estime de soi et à dépression. Pour Promouvoir des actions que font face à cette question, il est important de mettre en évidence la performance des psychologues. Cette étude a eu l'objectif de comprendre la signification d'un soutien psychologique aux femmes dans les situations de violence domestique dans le contexte de la Stratégie de la santé de la famille. Des Entrevues ont été faites avec 52 professionnels qui travaillent dans les établissements de santé à Santa Catatina, une vile au sud du Brésil . La collecte et l'analyse des données ont été basées sur la théorie enracinée. Les Professionnels de la santé croient que les femmes qui ont passé pour une expérience de violence domestique ont besoin de soutien psychologique et ils leur ont orientées au psychologue, par penser que ce professionnel est mieux préparé pour habiliter les femmes afin de rompre avec la violence . Cependant, ils se plaignent du nombre limité de psychologues compte tenu de la demande de la communauté. Donc, cette étude souligne pour la nécessité de repenser les actions de santé dans le contexte des soins primaires, de l'intégration et des processus intersectoriels afin d’assurrer que les femmes aient le soutien psychologique indispensable pour affronter la violence domestique.

Mots-clés: violence contre les femmes, violence familiale, santé de la familie, assistance intégrale à la sante des femmes, psychologie. 


\section{Atención a mujeres en situación de violencia de género: Importancia del psicólogo en la Estrategia de Salud de la Familia}

Resumen: La violencia doméstica pone en peligro la salud mental de las mujeres y se asocia con una baja autoestima y depresión. En la promoción de acciones para hacer frente a esta lesión, es el trabajo de los psicólogos. Este estudio tuvo como objetivo comprender el significado del apoyo psicológico a las mujeres en situaciones de violencia doméstica dentro de la Estrategia de Salud de la Familia. Las entrevistas se celebraron con los 52 profesionales que trabajan en centros de salud en Santa Catatina, Brasil. La recopilación y análisis de los datos se basan en la Teoría Fundamentada. Profesionales de la salud significán que las mujeres que sufren de violencia doméstica necesitan apoyo psicológico y se refieren a la psicóloga, considerándolos mejor preparados para el empoderamiento de las mujeres con el fin de romper con la violencia. Sin embargo, se quejan del limitado número de psicólogos, teniendo en cuenta la demanda de la comunidad. Las señales a la necesidad de replantear las acciones de salud en el contexto de la Atención Primaria de la Salud, de la integración y los procesos intersectoriales para que las mujeres necesitan apoyo psicológico para hacer frente a la violencia doméstica está asegurada.

Palabras clave: violencia contra las mujeres, violencia en la familia, salud familiar, psicología.

\section{Referências}

Barreiros, E. X., Camargo, C. L., Diniz, N. M. F., \& Gomes, N. P. (2011). Historia oral de homens que praticaram violência sexual contra crianças e adolescentes. Oralidades (USP), 9(5), 95-108.

Blanques, A. M. (2010). Um projeto de intervenção social visto pelos seus agentes: estudo psicossocial do Programa de Saúde da Família. Psicologia USP, 21(4), 809-831.

Blasco, J. C. (2000). Análisis del concepto "Dependencia Emocional. In I Congresso Virtual de Psiquiatria: Transtornos de Personalidade. Recuperado de http:// www.psiquiatria.com/congreso_old/mesas/mesa6/ conferencias/6_ci_a.htm

Brasil. (2006). Lei Maria da Penha. Lei n.11.340, de 07 de agosto de 2006. Brasília, DF. Autor. Recuperado de http://www. planalto.gov.br/ccivil/_ato2004-2006/2006/lei/111340.htm

Brasil. Ministério da Saúde. (2008). Temático de prevenção de violência e cultura de paz III. Brasília, DF: Autor.

Campos, G. W. S., \& Domitt, A. C. (2007). Apoio matricial e equipe de referência: uma metodologia para gestão de trabalho interdisciplinar em saúde. Caderno Saúde Pública, 23(2), 399-407.

Day, V. P. (2003). Violência doméstica e suas diferentes manifestações. Revista de Psiquiatria, 25(supl. 1), 9-21.

Dias, B. M. (2007). A Lei Maria da Penha na justiça: a efetividade da Lei 11.340/2006 de combate à violência doméstica e familiar contra a mulher. São Paulo, SP: Revista dos Tribunais.

D’oliveira, A. F. P. L. (2000). Violência de gênero, necessidades de saúde e uso de serviços em atenção primária (Tese de Doutorado). Faculdade de Medicina, Universidade de São Paulo, São Paulo.

Freire, F. M. S., \& Pichelli, A. A. W. S. (2013). O psicólogo apoiador matricial: percepções e práticas na atenção básica. Psicologia: Ciência e Profissão, 33(1), 162-173.

Gomes, N.P.(2009). Trilhando caminhos para onfrentamento da violência conjugal (Tese de Doutorado). Escola de Enfermagem, Universidade Federal da Bahia, Salvador.
Hanada, H., D’Oliveira, A. F. P. L., \& Schraiber, L. B. (2008). Os psicólogos e a assistência a mulheres em situação de violência. Fazendo Gênero 8: Corpo, Violência e Poder. Recuperado de http://www.fazendogenero.ufsc.br/8/st33.html

Hirigoyen, M. F. (2006). Os homens violentos. In M. F. Hirigoyen, A violência no casal: da coação psicológica à agressão física (M. H. Kühner, trad., pp. 121-136) Rio de Janeiro, RJ: Bertrand Brasil.

Kiss, L. (2004). Temas médico-sociais e a intervenção em saúde: a violência contra mulheres no discurso dos profissionais (Dissertação de Mestrado). Faculdade de Medicina, Universidade de São Paulo, São Paulo.

Maia, C. A. T., Mondaca, A. D. C. A., Lima, J. A., Colodo, M., Lemos, T. M., Rossi, A. S., \& Vieira, M. J. N. (2001). Mulheres vitima de violência. Recuperado de http://www.aads.org.br/rhamas/vitimas.html

Nepomuceno, L. B., \& Brandão, I. R. (2011). Psicólogos na estratégia saúde da família: caminhos percorridos e desafios a superar. Psicologia: Ciência e Profissão, 31(4), $762-777$.

Pedrosa, C. M. (2003). Mulheres em situação de violência: o discurso dos profissionais de saúde (Dissertação de Mestrado). Programa de Psicologia Social, Universidade Católica de São Paulo, São Paulo.

Redes Humanizadas de Atendimento às Mulheres Agredidas Sexualmente. (2001). Mulheres vitimas de violência. São Paulo, SP: Autor. Recuperado de http://www.ipas. org.br/rhamas/index.html

Rose, N. (2008). Psicologia como uma ciência social. Revista Psicologia \& Sociedade, 20(2), 155-164.

Schraiber, L. B., D’oliveira, A. F. P. L., França-Junior, I., \& Pinho, A. (2002). Violência contra a mulher: estudo de uma unidade de atenção primária à saúde. Revista de Saúde Pública, 36(4), 470-477.

Silva, M. A., Falbo Neto, G. H., \& Cabral Filho, J. E. (2009). Maus tratos na infância de mulheres vítimas de violência. Psicologia em Estudo, 14(1), 121-127. 
Strauss, A. L., \& Corbin, J. M. (2008). Pesquisa qualitativa: técnicas e procedimentos para o desenvolvimento de teoria fundamentada. Porto Alegre, RS: Artmed.

Stromquist, N. P. (2005). Comments on EPT global monitoring. Ti o $n, 26(5)$.
Waiselfisz, J. J.(2012). Mapa da Violência 2012. Os novos padrões da violência homicida no Brasil. São Paulo, SP: Instituto Sangari. Recuperado de http://www. mapadaviolencia.org.br/pdf2012/mapa2012_mulher.pdf

Recebido: $13 / 12 / 2012$

$1^{a}$ revisão: 29/12/2013

Aceito: 17/01/2014 
